\title{
Antifungal, Contraceptive, Anti-Cancer, Mosquito Repellent Properties of Azadirachta Indica: A review
}

\author{
Article by Niharika Anand \\ Doctor of Medicine (MD1), Texila American University, Guyana, South America \\ E-mail:niha1798@gmail.com
}

\begin{abstract}
Azadirachta indica, or more commonly known as Neem, is noted for a variety of medicinal properties. Large numbers of unique phytochemical constituents have been purified from this plant that are being used effectively as anti-fungal, anti-bacterial and anti-inflammatory agents. Neem leaf is an essential ingredient of many Ayurveda medicines. Gedunin and nimbidol are found in the leaves of neem, are shown powerful antifungal activity in various fungal infections in humans. Traditional forms of medicine practiced for centuries in Africa and Asia has been reported that, the leaf extract of Azardica indica possess wound healing activity when applied over external wound and it also taken internally to boost immunity and improve overall health. Several parts of this plant are used in many ailments such as intestinal pain, diabetics, fever, hepatitis, etc. Although the effects of neem are widely known and appreciated, thus far steps haven't been taken to study its individual properties, and prove through research the extent of its prowess. This review gives a bird's eye view mainly on the some of the biological activity of the neem includes antifungal, contraceptive and anti-cancer and mosquito repellent properties.
\end{abstract}

Keywords: Azardica indica, antifungal, contraceptive, anti-cancer, P.ovale, mosquito repellent.

\section{Introduction}

Azadirachta indica or Neem, is one of the most versatile medicinal plant having a wide spectrum of biological activity and has become a cynosure of modern medicine. Because of number of biological activities, it is widely used for the treatment of several diseases in India and its neighboring countries. Epidemiological observations, though inconclusive, are suggestive that Neem consumption may reduce the risk of many disease including cancer in humans (Houghton et al., 1995). Every part of the tree has been used as a household remedy against various human ailments and it is the one of the most revenue releasing plant because of several pharmacological activities associated with it. The present review mainly highlights on antifungal, contraceptive and anti-cancer and mosquito repellent properties of Azadirachta indica.

\section{Various biological properties of azadirachta indica}

\section{Antifungal property}

Generally, in developing countries, most of the skin infections are especially associated with fungal infections (Ping et al., 2007) and last two decades millions of people are affected with fungal infections. (Wong et al., 1994). Currently available antifungal drugs are less effective and induce many side effects. Therefore, it is necessary to search for more effective and less toxic novel antifungal agents that would overcome these disadvantages. Interestingly, plants are widely employed in folk medicine, mainly in communities with inadequate conditions of public health and sanitation.

Fungicidal properties of neem extracts are promising and significantly reduced conidial germination in several fungi. The extracts of Azadirachta indica from bark and leaf, inhibited both spore germination and mycelial growth of Epidermophyton floccosum, Microsporum gypseum and Trichophyton mentagrophyte (Dube et al., 1987). Leaf extracts of neem were found to have a potent antidermatophytic activity against T.rubrum, T. violaceum, M. nanum and E. floccosum (Okemo et al., 2001).

Dandruff a very common fungal scalp disorder that affects $50 \%$ of the population, mainly between ages 15-50. Most of the commercially available anti-dandruff hair shampoos contain some form of antifungal agents that appear to reduce the incidence of the disease. There are no good scientific studies 
done to prove the antifungal activity of commercially available hair shampoos. Although many antidandruff shampoos are available in the market, most are made of chemicals that may harm the normal human cells and the environment.

A study done by Niharika et al., (2010) demonstrated the effectiveness of different concentrations of neem leaf extract against Pityrosporum ovale, a main causative organism of dandruff. To determine this effect, she has selected neem leaves and macerated in $400 \mathrm{~mL}$ of ethanol and covered with parafilm. It was allowed to stand in room temperature for 48 hours then it was filtered. To get $100 \%$ extract, an amount of the extract was placed in an evaporating dish to be subjected to water-bath. The extract looked dark brown in its liquid form. The fungus (P. ovale) was cultured in the Laboratory. Various levels of Neem extract concentration $(25 \%, 50 \%, 75 \%$, and $100 \%)$ were prepared. The inhibiting capacity of each level on fungus that causes dandruff was tested using agar cup method. To establish a firmer and more solid foundation of the contention that high antifungal properties is present, experiment was conducted in three treatments. Although all concentration showed inhibition of the fungus, the $100 \%$ extract of neem leaves produced the widest zone of inhibition which was found statistically higher than the other concentration levels. This study proves the effectiveness of need against P.ovale, a fungus that causes dandruff (Niharika et al., 2010)

Natarajan et al., (2002) found the effectiveness of neem leaf and seed extract on inhibition of dermatophytes, which include three genera of fungi that commonly cause skin disease of people and animals. In this study, he tested the antidermatophytic activity of leaf and seed extract of Azadirachta indica on Trichophyton ruberum, Trichophyton Mentagrophytes, Trichophyton violaceum, Microsporum nanum and Epidermophyton floccosum.

In a recent in-vitro study, aqueous, ethanolic and ethyl acetate fractions of neem leaves have shown the inhibition of some fungal pathogens such as Aspergillus flavus, Aspergillus fumigatus, Aspergillus niger, Aspergillus terreus, Candida albicans and Microsporum gypseum (Mahmoud et al., 2011). Among three fractions, 20\% ethyl acetate fraction has shown the highest inhibition of aforementioned fungal pathogens when compared to the same concentration of the other extracts. Further, High Performance Liquid Chromatography (HPLC) and Magnetic Resonance (NMR) spectroscopic analysis found nimonol that is the active phytoconstituents responsible for fungicidal action of ethyl acetate fraction of neem.

\section{Antifertility activity}

Oral contraceptives are an effective means of preventing pregnancy. However, use of contraceptives has been associated with a variety of physical and psychological side effects includes, venous thromboembolism (Kemmeren et al., 2002), weight gain, high blood pressure (Fisch et al., 1977) and cervical cancer (Moreno et al., 2002). .

Several previous studies have shown the antifertility activity of neem seed oil (NSO) and leaf extracts in men. It significantly prevented spermatogenesis, decreased sperm mobility, count and cessation of fertility with producing loss of libido or potency. Furthermore, NSO possess abortifacient and antiimplantation properties. The Neem oil extract shows the spermicidal activity against human and rhesus monkey were found by Sinha et al (1984). Vaginal biopsy reveals no side effect, while the studies of radio-isotope which indicate non-antiovulatory and non-absorption in the vagina (Sharma et al., 1959). These research enabled neem oil formulation "sensal" which is used as a powerful contraceptive in India [Kabeh et al., 2007].

\section{Anticancer properties of neem}

Cancer continues to be an enigmatic challenge for cancer biologists and medical practitioners. Several tantalizing claims for discovering a sure cure for cancer have been made by scientists from time to time; yet a dependable cure against most cancers remains a challenge even today. One of the primary reasons for this is the multiple pathways of survival adopted by cancer cells. Blocking a few pathways of their survival does not ensure their targeted elimination. That is why researchers are now trying to target them through multiple pathways with minimum possible side effects and discomfort to patients. In recent years, certain herbal products and ethnomedicines have drawn keen attention of researchers 
and medical practitioners primarily because of convincing anticancer properties with negligible unpleasant side effects and discomfort to patient.

During the last decade, the anticancer properties of neem have been investigated in detail from several scientific angles. The active ingredients obtained from the plant have been demonstrated unequivocally to induce apoptosis in several types of tumor cells and to organize and gear up the immune system to take on the cancer cells through cross priming. Regular use of neem and its preparations have been found to prevent onset of cancer through multiple mechanisms including free radical scavenging, carcinogen-detoxification, DNA repair, cell cycle alteration, programmed cell death (apoptosis) and autophagy, immune surveillance, anti-inflammatory, anti-angiogenic, anti-invasive and anti-metastatic activities as well as their ability to modulate several dysregulated oncogenic signalling pathways (Paul et al., 2005) Against a wide variety of human cancer cell lines and animal models for human cancers including colon, stomach, Ehrlichs carcinoma, lung, liver, skin, oral, prostate and breast cancers; neem products have been demonstrated to produce impressive anticancer results.

Pre-clinical research work done during the last decade has fine-tuned our understanding of the anticancer properties of the crude and purified products from this plant. The anticancer properties of the plant have been studied largely in terms of its preventive, protective, tumor-suppressive, immunomodulatory and apoptotic effects against various types of cancer and their molecular mechanisms. The cogent data on the anticancer biology of products from A. indica deserve multiinstitutional clinical trials as early as possible. The prospects of relatively cheaper cancer drugs could then be brighter, particularly for the under-privileged cancer patients of the world (Patel et al., 2016).

\section{Neem as mosquito repellent}

The mosquitoes are a serious threat to public they are known vector of many life-threatening diseases like malaria, filariasis and dengue. Most of the commercial repellents are prepared by Dimethyl Phthalate (DMP), Allethrin and N - diethyl - 3 - methylbenamide. These are effective in killing many mosquitoes, however, they are high cost and not safe for long term use.

In search of alternative and protection from mosquito bites, neem has found to be effective mosquito repellents. (Nagpal et al., 2001). A number of studies have shown that Neem compounds are more effective insect repellent than the widely used synthetic chemical N, N-diethylm- tolumide (Farahna et al, 2010). Azadirachta indica and its derived products have shown a variety of insecticidal properties. A recent research discusses the larvicidal activity of neem-based biopesticide for the control of mosquitoes (Dua et al., 2009).

Carbondioxide, excretory products and lactic acid present in sweat of humans attracts the female mosquitoes. Usually, the female mosquitos precepts the odor through chemoreceptors present in the antennae of mosquitoes. Most of the repellents block the lactic acid receptors thus destroying upwind flight and as a result, the mosquito lose contact with the host (Patel et al., 2012). Additionally, mosquito repellants also work by masking human scent or by using a scent which insects naturally avoid. The presence of the odor in the neem seed and kernel oil must have contributed to the repellent action. Taste molecules are also involved; this suggested that the repellent deters biting and feeding because it activates taste cells that are present on the insect's tongue, legs and wing margin. When mosquito lands, it tastes the skin of the host with it gustatory receptors before it bites, thus there is avoidance responses not only through the sense of smell but also through the sense of taste. Neem seed and kernel have a punget smell which is similar to garlic and it contain sulfurous compound as reported by Akin, (2010), this attribute to its reduce plaque and act as mosquito repellent. (Akin et al., 2010)

The neem oil formulation was found effective in controlling mosquito larvae in different breeding sites under natural field conditions. As neem trees are widely distributed in India, their formulations may prove to be an effective and eco-friendly larvicide, which could be used as an alternative for malaria control.

\section{Conclusion}

Azadirachta indica has attracted worldwide prominence in recent years, owing to its wide range of medicinal properties. Neem has been extensively used in Ayurveda, Unani and Homoeopathic medicine and has become a cynosure of modern medicine. Neem elaborates a vast array of biologically active 
compounds that are chemically diverse and structurally complex. More than 140 compounds have been isolated from different parts of neem. All parts of the neem tree- leaves, flowers, seeds, fruits, roots and bark have been used traditionally for the treatment of inflammation, infections, fever, skin diseases and dental disorders. The medicinal utilities have been described especially for neem leaf. Neem leaf and its constituents have been demonstrated to exhibit immunomodulator, anti-inflammatory, antihyperglycaemic, antiulcer, antimalarial, antifungal, antibacterial, antiviral, antioxidant, antimutagenic and anticarcinogenic properties. Extensive research in the area of isolation and characterization of the active principles of neem plant is essential so that better, safer and cost effective drugs for curing various diseases and infections can be developed. In spite of its numerous benefits, there are only a handful of studies exploring the medicinal aspects of neem. Further research can be done to propagate neem in the pharmacological and medical field.

\section{Acknowledgement}

I hereby acknowledge Dr. Jagan Nadipelly for his guidance in writing this article and for reviewing it before publication.

\section{References}

[1]. Akin M., (2010) Neem (Azadirachta indica) Natural Standard, www.natural standard.com.

[2]. Dua, Virendra K.; Pandey, Akhilesh C.; Raghavendra, Kamaraju; Gupta, Ashish; Sharma, Trilochan; Dash, Aditya P. (2009) Larvicidal activity of neem oil (Azadirachta indica) formulation against mosquitoes Malaria Journal.

[3]. Dube S, Tripathi S. (1987) Toxicity of some plants against dermatophytes. National Academy of Sciences, India, Science Letters, 10(2): 45-48.

[4]. Farahna, M., S. Bedri, S. Khalid, M. Idris, C.R. Pillai and E.A. Khalil (2010). Anti-plasmodial effects of Azadirachta indica in experimental cerebral malaria: Apoptosis of cerebellar Purkinje cells of mice as a marker. N. Am. J. Med. Sci., 2: 518-525.

[5]. Fisch I. R., and Frank J. (1977). Oral contraceptives and blood pressure. The Journal of the American Medical Association, 237, 2499-2503.

[6]. Houghton PJ. (1995). The role of plants in traditional medicine and current therapy. J Alter Complement Med, 1:131-43.

[7]. Kabeh JD and M.G.D.S.S. Jalingo (2007) Mini review exploiting neem for improved life / Int J agribiol; vol 9, no.3.

[8]. Kemmeren J. M., Tanis B. C., van den Bosch M. A. A. J., Bollen E. L. E. M., Helmerhorst F. M., van der Graaf Y. and Algra A. (2002). Risk of arterial thrombosis in relation to oral contraceptives (RATIO) study: Oral contraceptives and the risk of ischemic stroke. Stroke, 33, 1202-1208.

[9]. Khillare, B., Shrivastav T.G et al.(2003) Spermicidal activity of Azadirachta indica (neem) leaf extract Contraception Journal , Volume 68, Issue 3, 225 - 229

[10]. Mahmoud, D. A., Hassanein, N. M., Youssef, K. A., \& Abou Zeid, M. A. (2011). Antifungal activity of different neem leaf extracts and the nimonol against some important human pathogens. Brazilian Journal of Microbiology, 42(3), 1007-1016.

[11]. Moreno V., Bosch F. X., Muñoz N., Meijer C. J. L. M., Shah K. V. and Walboomers J. M. M., . (2002). Effect of oral contraceptives on risk of cervical cancer in women with human papillomavirus infection: The IARC multicentric case-control study. The Lancet, 359, 1085-1092.

[12]. Nagpal BN, Srivastava A, Valecha N, Sharma VP, (2001). Repellent action of neem cream against Anopheles culicifacies and Culex quinquefasciatus. Curr Sci; 80: 1270-1.

[13]. Natarajan V, Pushkala S, Karuppiah VP, Prasad PV. (2002) Anti dermatophytic activity of Azardirachta indica (neem) by invitro study. Indian J Pathol Microbiol. Jul; 45(3):311-3.

[14]. Niharika Anand, Arulsamy Anand, Johnson M. Aquicio. (2010) Antifungal properties of neem (azardirachta indica) leaves extract to treat hair dandruff E-International Scientific Research Journal ISSN: 2094-1749 Volume: 2 Issue: 3.

[15]. Okemo PO, Mwatha WE, Chabrab SC, Fabryc W. (2001). The kill kinetics of Azadirachtaindica A. juss. (Meliacae) extracts on Staphylococcus aureus, Escherichia coli, Pseudomonas aeruginosa and Candida albicans.

Afr J Sci Technol, 2:113-118 
[16]. Patel EK., Gupta A., and Oswai RJ. (2012) A Review On: Mosquito Repellent Method. IJPCBS, 2(3), 310317

[17]. Patel SM, Nagulapalli Venkata KC, Bhattacharyya P, Sethi G, Bishayee A. (2016) Potential of neem (Azadirachta indica L.) for prevention and treatment of oncologic diseases. Epubmed 2016 Mar 24.

[18]. Paul R1, Prasad M, Sah NK (April 2005) Anticancer biology of Azadirachta indica L (neem): a mini review Current Medicinal Chemistry - Anti-Cancer Agents 5(2):149-6.

[19]. Ping-Hsein, Chi-Weilee, Jia-Ying Chou, Murugan M, Bor-Jinn Shieh, Hueih-Mir Chen.(2007) Antifungal activity of crude extracts and essential oil of Moringa oleifera L. Bioresource Technology, 98: 232-236.

[20]. Sharma V N, Saxena K P. (1959) Spermicidal action of sodium nimbinate Indian journal of medical research, 47, 322-324.

[21]. Subapriya R1, Nagini S. (2005) Medicinal properties of neem leaves: a review. Current Medicinal Chemistry Anticancer Agents. Mar; 5(2):149-6.

[22]. Wong B, Klei B, Kozel T. (1994) Immunologic approaches and metabolite detection. The second NIAID Workshop in Medical Mycology, University of Arizona, Northern Arizona University, Flagstaff, AZ, June 8-11. 